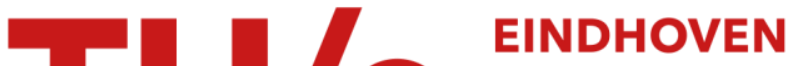 UNIVERSITY OF TECHNOLOGY
}

\section{Adaptive Image Restoration Based on Local Robust Blur Estimation}

\section{Citation for published version (APA):}

Hu, H., \& Haan, de, G. (2007). Adaptive Image Restoration Based on Local Robust Blur Estimation. In J. BlancTalon, \& W. Philips (Eds.), Proceedings of the 9th international conference on Advanced Concepts for Intelligent Vision Systems (ACIVS 2007) 28-31 August 2007, Delft, The Netherlands (pp. 461-472). (Lecture Notes in Computer Science; Vol. 4678). Springer. https://doi.org/10.1007/978-3-540-74607-2_42

DOI:

10.1007/978-3-540-74607-2_42

Document status and date:

Published: 01/01/2007

\section{Document Version:}

Publisher's PDF, also known as Version of Record (includes final page, issue and volume numbers)

\section{Please check the document version of this publication:}

- A submitted manuscript is the version of the article upon submission and before peer-review. There can be important differences between the submitted version and the official published version of record. People interested in the research are advised to contact the author for the final version of the publication, or visit the $\mathrm{DOI}$ to the publisher's website.

- The final author version and the galley proof are versions of the publication after peer review.

- The final published version features the final layout of the paper including the volume, issue and page numbers.

Link to publication

\section{General rights}

Copyright and moral rights for the publications made accessible in the public portal are retained by the authors and/or other copyright owners and it is a condition of accessing publications that users recognise and abide by the legal requirements associated with these rights.

- Users may download and print one copy of any publication from the public portal for the purpose of private study or research.

- You may not further distribute the material or use it for any profit-making activity or commercial gain

- You may freely distribute the URL identifying the publication in the public portal.

If the publication is distributed under the terms of Article 25fa of the Dutch Copyright Act, indicated by the "Taverne" license above, please follow below link for the End User Agreement:

www.tue.nl/taverne

Take down policy

If you believe that this document breaches copyright please contact us at:

openaccess@tue.nl

providing details and we will investigate your claim. 


\title{
Adaptive Image Restoration Based on Local Robust Blur Estimation
}

\author{
Hao $\mathrm{Hu}^{1}$ and Gerard de $\operatorname{Haan}^{1,2}$ \\ ${ }^{1}$ Eindhoven University of Technology, Den Dolech 2, \\ 5600 MB Eindhoven, The Netherlands \\ ${ }^{2}$ Philips Research Laboratories, High Tech Campus 36, \\ 5656 AE Eindhoven, The Netherlands
}

\begin{abstract}
This paper presents a novel non-iterative method to restore the out-of-focus part of an image. The proposed method first applies a robust local blur estimation to obtain a blur map of the image. The estimation uses the maximum of difference ratio between the original image and its two digitally re-blurred versions to estimate the local blur radius. Then adaptive least mean square filters based on the local blur radius and the image structure are applied to restore the image and to eliminate the sensor noise. Experimental results have shown that despite its low complexity the proposed method has a good performance at reducing spatially varying blur.
\end{abstract}

\section{Introduction}

Focal blur, or out-of-focus blur in images and videos occurs when objects in the scene are placed outside the focal plane of the camera. Due to a limited focal range of optical lenses or sub-optimal settings of the camera, the resulting image may suffer from blur degradation. As objects with varying distance are often differently blurred in the image, accurate blur estimation is essential for image restoration. The technique to estimate the blur and restore all-in-focus images is called multi-focusing. The demand for such a technique is emerging in many applications, such as digital camera and video surveillance. The technique potentially enables the use of algorithms running on relatively cheap DSP chips instead of expensive optical parts.

Many techniques have been proposed to restore the original image from the blurred image. Most of them, like [1, are designed to estimate the spatially invariant blur. For local blur estimation, methods are typically based on an analysis of an ideal edge signal. In Elder's method [2] the blurred edge signal is convolved with the second derivative of Gaussian function and the response has a positive and a negative peak. The distance between these peak positions can be used to determine the blur radius. Another approach from Kim [3] is based on an isotropic discrete point spread function (PSF) model. The one-dimensional step response along the orthogonal direction of edge direction will be estimated and the PSF can be obtained by solving a set of linear equations related to 
the step response. Both Elder's and Kim's method require detection of the edge direction, which adds complexity to the algorithm.

In this paper, we propose a new multi-focusing method that features low complexity aiming at real-time implementation. The proposed method adopts a simple non-iterative blur estimator, as proposed in our earlier work [4. The blur estimator uses a Gaussian isotropic PSF model and the difference between digitally re-blurred versions of an image is used to estimate the blur radius without edge detection. As a de-blurring filter typically has a high pass characteristic, the sensor noise may be amplified during the restoration process. To avoid this and even suppress the noise, adaptive filters based on the local blur radius and image structure information [5] are applied in the image restoration part.

The rest of the paper is organized as follows. In Section 2 we present the proposed blur estimation algorithm and its analysis based on an ideal edge model. Section 3 shows the proposed adaptive image restoration using local image structure and blur radius. Some experimental results on natural images are provided in Section 4 and, finally, Section 5 concludes the paper.

\section{Local Blur Estimation}

We analyse the blur estimation with a one dimensional (1D) signal. We assume an ideal edge signal and a discrete Gaussian blur kernel. The edge is modeled as a step function with amplitude $A$ and offset $B$. For a discrete signal, the edge $f(x)$ shown in Fig. 1 is

$$
f(x)=\left\{\begin{array}{ll}
A+B, & x \geq 0 \\
B, & x<0
\end{array}, x \in \mathbb{Z}\right.
$$

where $x$ is the position. The focal blur kernel is modeled by a discrete Gaussian function:

$$
g(n, \sigma)=C(\sigma) \exp \left(-\frac{n^{2}}{2 \sigma^{2}}\right), n \in \mathbb{Z}
$$

where $\sigma$ is the unknown blur radius to be estimated and $C(\sigma)$ is the normalization factor. The normalization implies:

$$
\sum_{n \in \mathbb{Z}} g(n, \sigma)=\sum_{n \in \mathbb{Z}} C(\sigma) \exp \left(-\frac{n^{2}}{2 \sigma^{2}}\right)=1
$$

$C(\sigma)$ admits no closed form expression, but the approximation $\frac{1}{\sqrt{2 \pi} \sigma}$ can be considered acceptable when $\sigma>0.5$. Then the blurred edge $b(x)$ will be:

$$
\begin{aligned}
b(x)= & \sum_{n \in \mathbb{Z}} f(x-n) g(n, \sigma) \\
= & \left\{\begin{array}{l}
\frac{A}{2}\left(1+\sum_{n=-x}^{x} g(n, \sigma)\right)+B, \quad x \geq 0 \\
\frac{A}{2}\left(1-\sum_{n=x+1}^{-x-1} g(n, \sigma)\right)+B, x<0
\end{array}, x \in \mathbb{Z}\right.
\end{aligned}
$$




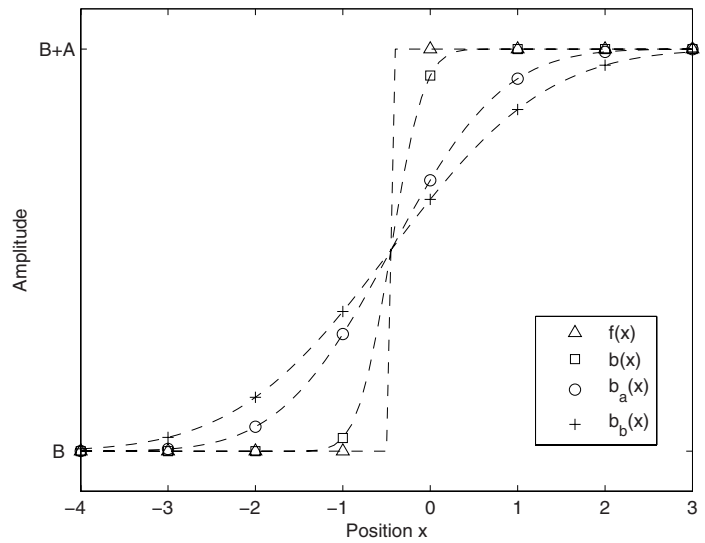

Fig. 1. The step edge $f(x)$, the blurred edge $b(x)$ and its two re-blurred versions $b_{a}(x)$, $b_{b}(x)$

As the convolution of two Gaussian functions with blur radii $\sigma_{1}, \sigma_{2}$ is:

$$
g\left(n, \sigma_{1}\right) * g\left(n, \sigma_{2}\right)=g\left(n, \sqrt{\sigma_{1}^{2}+\sigma_{2}^{2}}\right)
$$

re-blurring the blurred edge using Gaussian blur kernels with blur radius $\sigma_{a}$ and $\sigma_{b}\left(\sigma_{b}>\sigma_{a}\right)$, results in two re-blurred versions $b_{a}(x)$ and $b_{b}(x)$ :

$$
\begin{aligned}
& b_{a}(x)=\left\{\begin{array}{l}
\frac{A}{2}\left(1+\sum_{n=-x}^{x} g\left(n, \sqrt{\sigma^{2}+\sigma_{a}^{2}}\right)\right)+B, x \geq 0 \\
\frac{A}{2}\left(1-\sum_{n=x+1}^{-x-1} g\left(n, \sqrt{\sigma^{2}+\sigma_{a}^{2}}\right)\right)+B, x<0
\end{array}, x \in \mathbb{Z}\right. \\
& b_{b}(x)=\left\{\begin{array}{l}
\frac{A}{2}\left(1+\sum_{n=-x}^{x} g\left(n, \sqrt{\sigma^{2}+\sigma_{b}^{2}}\right)\right)+B, \quad x \geq 0 \\
\frac{A}{2}\left(1-\sum_{n=x+1}^{-x-1} g\left(n, \sqrt{\sigma^{2}+\sigma_{b}^{2}}\right)\right)+B, x<0
\end{array}, x \in \mathbb{Z}\right.
\end{aligned}
$$

To make the blur estimation independent of the amplitude and offset of edges, we calculate the ratio $r(x)$ of the differences between the original blurred edge and the two re-blurred versions for every position $x$ :

$$
r(x)=\frac{b(x)-b_{a}(x)}{b_{a}(x)-b_{b}(x)}
$$




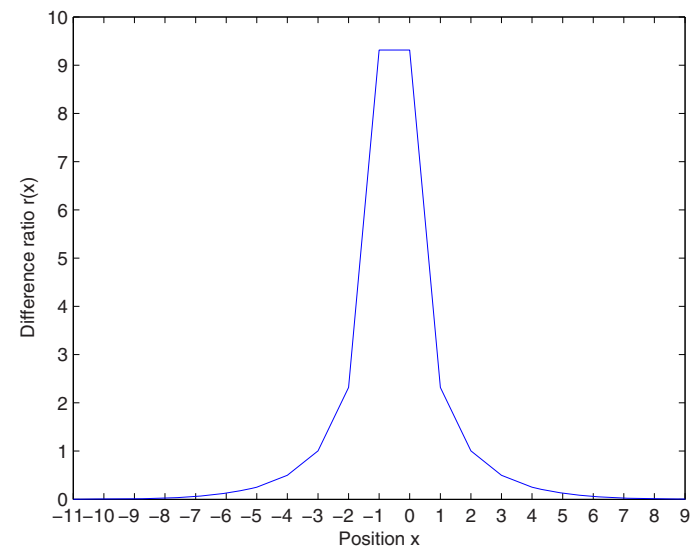

Fig. 2. Difference ratio among the edge

$$
=\left\{\begin{array}{l}
\frac{\sum_{n=-x}^{x}\left(g\left(n, \sqrt{\sigma^{2}+\sigma_{a}^{2}}\right)-g(n, \sigma)\right)}{\sum_{n=-x}^{x}\left(g\left(n, \sqrt{\sigma^{2}+\sigma_{b}^{2}}\right)-g\left(n, \sqrt{\sigma^{2}+\sigma_{a}^{2}}\right)\right)}, x \geq 0 \\
\sum_{n=x+1}^{-x-1}\left(g\left(n, \sqrt{\sigma^{2}+\sigma_{a}^{2}}\right)-g(n, \sigma)\right) \\
\sum_{n=x+1}^{-x-1}\left(g\left(n, \sqrt{\sigma^{2}+\sigma_{b}^{2}}\right)-g\left(n, \sqrt{\sigma^{2}+\sigma_{a}^{2}}\right)\right)
\end{array}\right.
$$

The difference ratio peaks at the edge position $x=-1$ and $x=0$ as shown in Fig. 2, So we obtain:

$$
r(x)_{\max }=r(-1)=r(0)=\frac{\frac{1}{\sigma}-\frac{1}{\sqrt{\sigma^{2}+\sigma_{a}^{2}}}}{\frac{1}{\sqrt{\sigma^{2}+\sigma_{a}^{2}}}-\frac{1}{\sqrt{\sigma^{2}+\sigma_{b}^{2}}}}
$$

When $\sigma_{a}, \sigma_{b} \gg \sigma$, we can use some approximations:

$$
\begin{aligned}
& \sqrt{\sigma^{2}+\sigma_{a}^{2}} \approx \sigma_{a} \\
& \sqrt{\sigma^{2}+\sigma_{b}^{2}} \approx \sigma_{b}
\end{aligned}
$$

which we use to simplify Equation 8:

$$
r(x)_{\max } \approx \frac{\frac{1}{\sigma}-\frac{1}{\sigma_{a}}}{\frac{1}{\sigma_{a}}-\frac{1}{\sigma_{b}}}=\frac{\left(\frac{\sigma_{a}}{\sigma}-1\right) \cdot \sigma_{b}}{\sigma_{b}-\sigma_{a}}
$$


or

$$
\sigma \approx \frac{\sigma_{a} \cdot \sigma_{b}}{\left(\sigma_{b}-\sigma_{a}\right) \cdot r(x)_{\max }+\sigma_{b}}
$$

Equation 9-11 shows that blur radius $\sigma$ can be calculated from the difference ratio maximum $r(x)_{\max }$ and re-blur radius $\sigma_{a}, \sigma_{b}$, independent of the edge amplitude $\mathrm{A}$ and offset $\mathrm{B}$. The identification of the local maximum of difference ratio $r(x)_{\max }$ not only estimates the blur radius, but also locates the edge position, which implies the blur estimation does not require a separate edge detection. This helps to keep the complexity low.

For the blur estimation in images, i.e. two dimensional (2D) signals, we use a 2D isotropic Gaussian blur kernel for the re-blurring. As any direction of an isotropic Gaussian function is a 1D Gaussian function, the proposed blur estimation is also applicable. Using 2D Gaussian kernels for the estimation avoids detecting the angle of the edge or gradient, as required in Elder's and Kim's method.

For simplicity, we implement the algorithm in a block-based manner to obtain a blur map of a natural image. A block size of $8 \times 8$ pixels has been used and we assign the blur radius to all pixels within the block. As shown in the block diagram in Fig 3. the difference ratios are calculated pixel-wise using the original image and its two re-blurred version. Then, in every block the maximum of the difference ratio is used to determine the blur radius in the block. To make the blur map smoother, we apply a post-processing using a minimum filter and a bicubic upscaling filter to obtain the final blur map on the pixel grid.

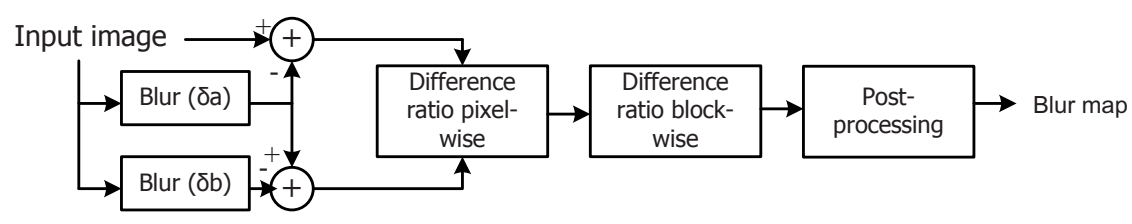

Fig. 3. The block diagram of the proposed algorithm

\section{Adaptive Image Restoration}

Many image restoration techniques [6] use an iterative approach to remove the blur, because they do not need to determine the inverse of a blur operator. However, for real-time applications, iterative approaches are less suitable. Therefore, we use LMS filters as an approximation to the inverse of the blur operation. In order to simultaneously restore the fine structure and eliminate the sensor noise, we adapt the LMS filters to a binary pattern classification of local image structure information and blur radius.

\subsection{Binary Pattern Classification}

Fine structure and sensor noise have distinguishable luminance patterns in natural images. We propose to use adaptive dynamic range coding (ADRC) 7] to 
classify local image structure. Within a local aperture in the image, the binary ADRC code of the pixels are defined as:

$$
\operatorname{ADRC}\left(x_{i}\right)=\left\{\begin{array}{l}
0, \text { if } x_{i}<\frac{x_{\max }+x_{\min }}{2} \\
1, \text { otherwise }
\end{array}\right.
$$

where $x_{i}$ is the value of pixels in the filter aperture and $x_{\max }, x_{\min }$ are the maximum and minimum pixel value in the filter aperture. One can see that the fine structures such as edges have regular patterns while the noise shows chaotic patterns.

To combine the blur radius into the classification, we quantize the local blur radius $\sigma$ obtained from the blur map into a binary $R B$ as:

$$
R B=\operatorname{round}\left(\frac{\sigma}{Q}\right)
$$

where $Q$ is predefined quantization step. The concatenation of ADRC code and $\mathrm{RB}$ gives the final binary classification code.

The diagram of the proposed adaptive restoration is shown in Fig. 4. The local image structure within a filter aperture centered at the output pixel is first classified by ADRC and local blur radius at the central pixel position. The LMS filter is used to calculate the output pixel with filter coefficients obtained from the look-up-table (LUT). The filter aperture slides pixel by pixel over the entire image. To avoid an impractical number of classes, we apply ADRC on pixels only in the central $3 \times 3$ aperture.

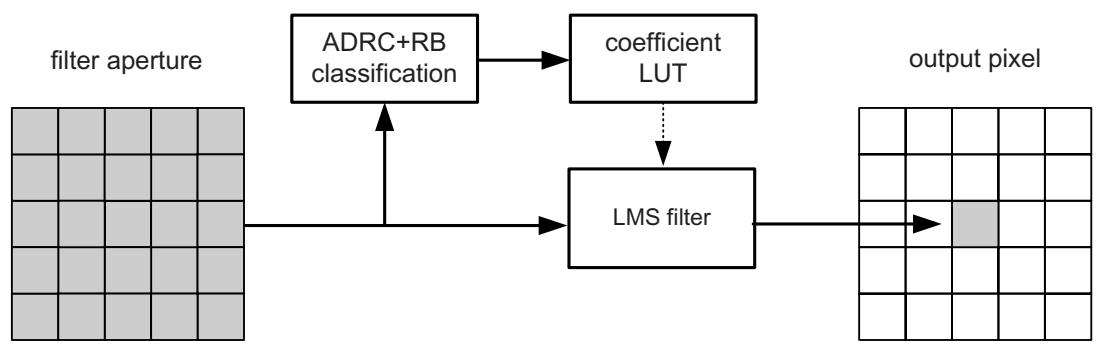

Fig. 4. The block diagram of the proposed algorithm

\subsection{Training Procedure}

The training procedure of the proposed method is shown in Fig. 5. To obtain the training set, we use the all-in-focus images as the reference output images. Furthermore we blur the original image with a Gaussian kernel with a range of blur radiuses and later add Gaussian noise to simulate sensor noise at an expected level. These blurred and corrupted versions of the original images are our simulated input images. Before training, the simulated input and the reference output pairs are collected pixel by pixel from the training material and are classified using ADRC and the blur radius on the input. The pairs that belong to 


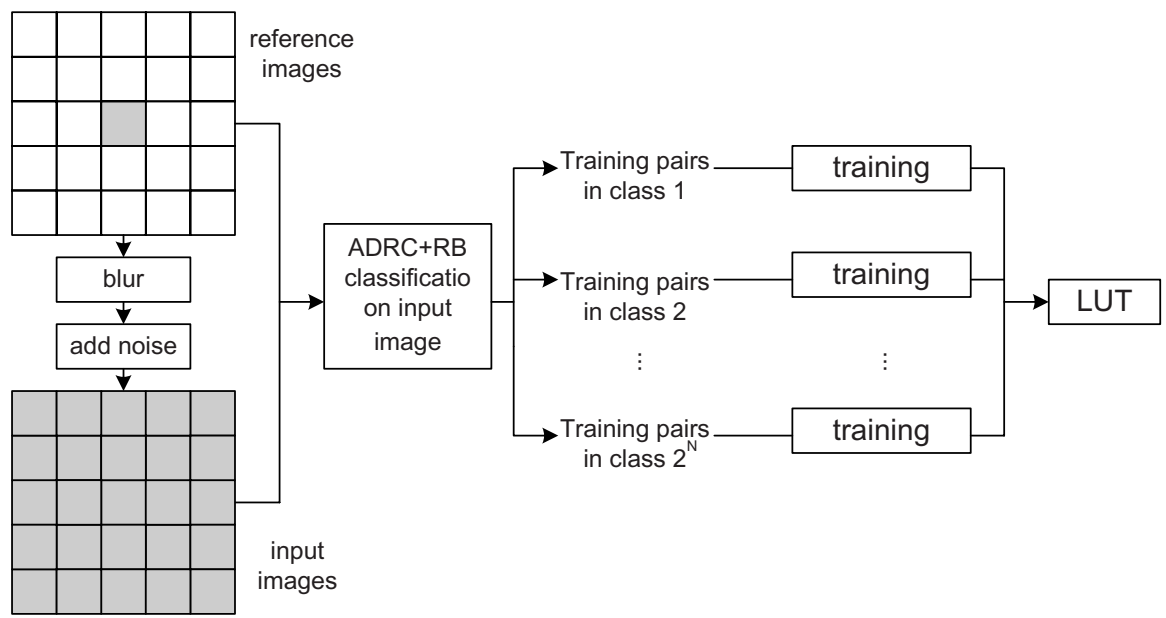

Fig. 5. The block diagram of the proposed algorithm

one specific class are used for the corresponding training, resulting in optimal filter coefficients for this class.

The optimal coefficients for each class are obtained by using the LMS algorithm. Suppose $X_{m}=\left[x_{1, m}, x_{2, m}, \ldots, x_{n, m}\right]^{T}$ is the input vector containing all the pixels in the filter aperture and within a class the total number of the input vectors $X_{1}, X_{2}, \ldots, X_{M}$ is $M$. Let $y_{m}$ be the reference output and $y_{m}^{h}$ be the output value of our adaptive filter for the input vector $X_{m}$. So we have:

$$
y_{m}^{h}=w_{1} x_{1, m}+w_{2} x_{2, m}+\ldots+w_{n} x_{n, m}
$$

where $W=\left[w_{1}, w_{2}, \ldots, w_{n}\right]^{T}$ are the filter coefficients.

The sum square error then is:

$$
e^{2}=\sum_{m=1}^{M}\left(y_{m}-y_{m}^{h}\right)^{2}
$$

Insert Equation 14 into Equation 15, then the summed square error becomes

$$
e^{2}=\sum_{m=1}^{M}\left[y_{m}-\left(w_{1} x_{1, m}+w_{2} x_{2, m}+\ldots+w_{n} x_{n, m}\right)\right]^{2}
$$

To get the minimal value of $e^{2}$, let the first derivatives of $e^{2}$ to $w_{1}, w_{2}, \ldots, w_{n}$ equal zero.

$$
\begin{aligned}
& \frac{\partial e^{2}}{\partial w_{1}}=\sum_{m=1}^{M} 2 x_{1, m}\left[y_{m}-\left(w_{1} x_{1, m}+\ldots+w_{n} x_{n, m}\right]=0\right. \\
& \ldots \\
& \frac{\partial e^{2}}{\partial w_{n}}=\sum_{m=1}^{M} 2 x_{n, m}\left[y_{m}-\left(w_{1} x_{1, m}+\ldots+w_{n} x_{n, m}\right)\right]=0
\end{aligned}
$$


Let

$$
\begin{aligned}
& X=\left[\begin{array}{ccc}
\sum_{m=1}^{m} x_{1, m} x_{1, m} & \ldots & \sum_{m=1}^{M} x_{1, m} x_{n, m} \\
\sum_{m=1}^{m} x_{2, m} x_{1, m} & \cdots & \sum_{m=1}^{M} x_{2, m} x_{n, m} \\
\vdots & \vdots \\
\sum_{m=1}^{M} x_{n, m} x_{1, m} & \cdots & \sum_{m=1}^{M} x_{n, m} x_{n, m}
\end{array}\right] \\
& Y=\left[\sum_{m=1}^{M} x_{1, m} y_{m}, \cdots, \sum_{m=1}^{M} x_{n, m} y_{m}\right]^{T}
\end{aligned}
$$

Equation 17 can be transformed into:

$$
X \cdot W=Y
$$

Please note that $X$ is the sum of the correlation matrices of the vectors $X_{1}, X_{2}, \ldots, X_{M}$. Then the coefficients $W$ can be solved by matrix inversion:

$$
W=X^{-1} \cdot Y
$$

\section{Experimental Results}

To demonstrate the performance of our proposed method, we used a natural image taken by a consumer digital camera as shown in Fig. 6, which is not included in the training images. The image shows three objects that are differently blurred. The restored image is shown in Fig. 7. The focus has been brought back to those differently blurred objects by the proposed adaptive restoration. Fig 8 shows the blur map estimated by the proposed method. In the blur map the lighter areas indicate a larger blur radius, while the darker areas indicate a smaller blur radius. One can see that different blur level can be clearly discriminated. In the blur estimation, the blur radius for the re-blurring kernels are $\sigma_{a}=1$ pixel, $\sigma_{b}=3$ pixels.

In order to show the effectiveness of our proposed adaptive image restoration method, we compare it with LMS filters which depend on local blur radius only and are trained with and without added noise respectively. Fig 10 shows image fragments from the test image processed by LMS filters with different settings. The LMS filters trained without added noise can reduce the blur, but they also amplify the noise. The proposed method not only suppresses the noise but also reduce the blur better due to the adaptivity to image structure, compared with LMS filters which depend on blur radius only. 


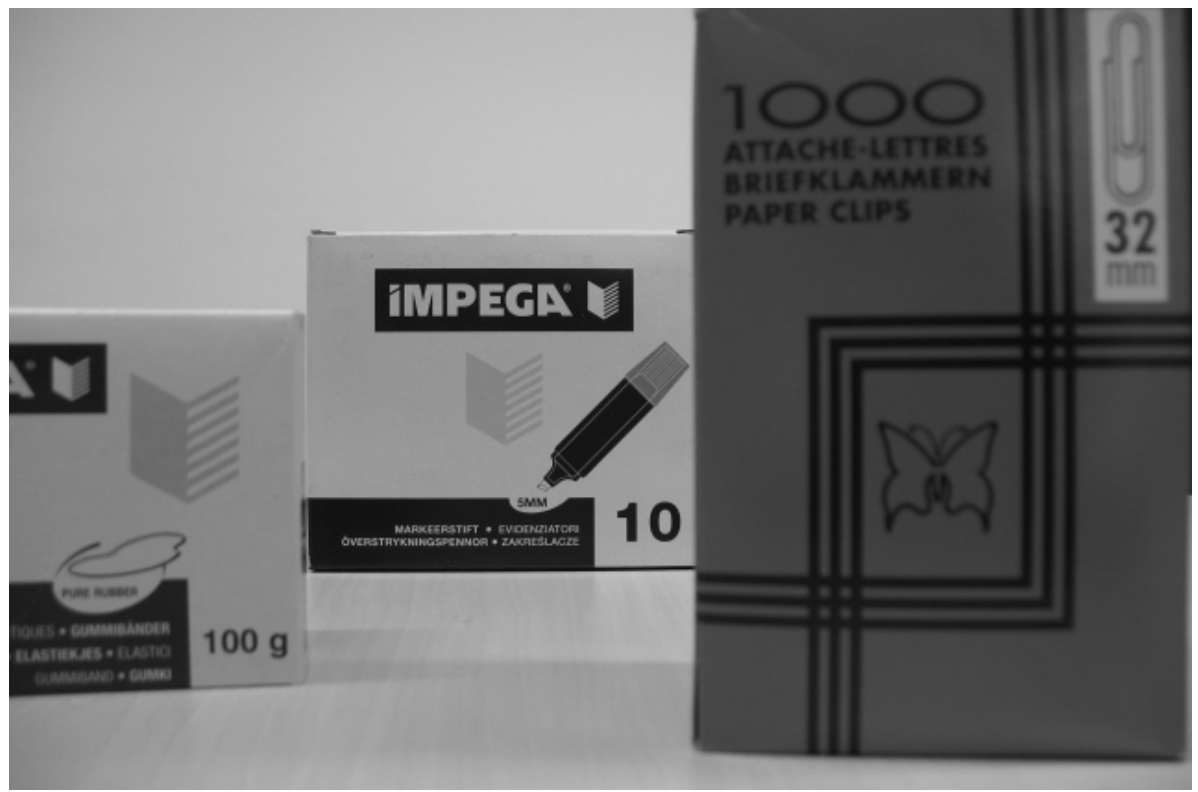

Fig. 6. The test image with differently blurred objects taken by a digital camera

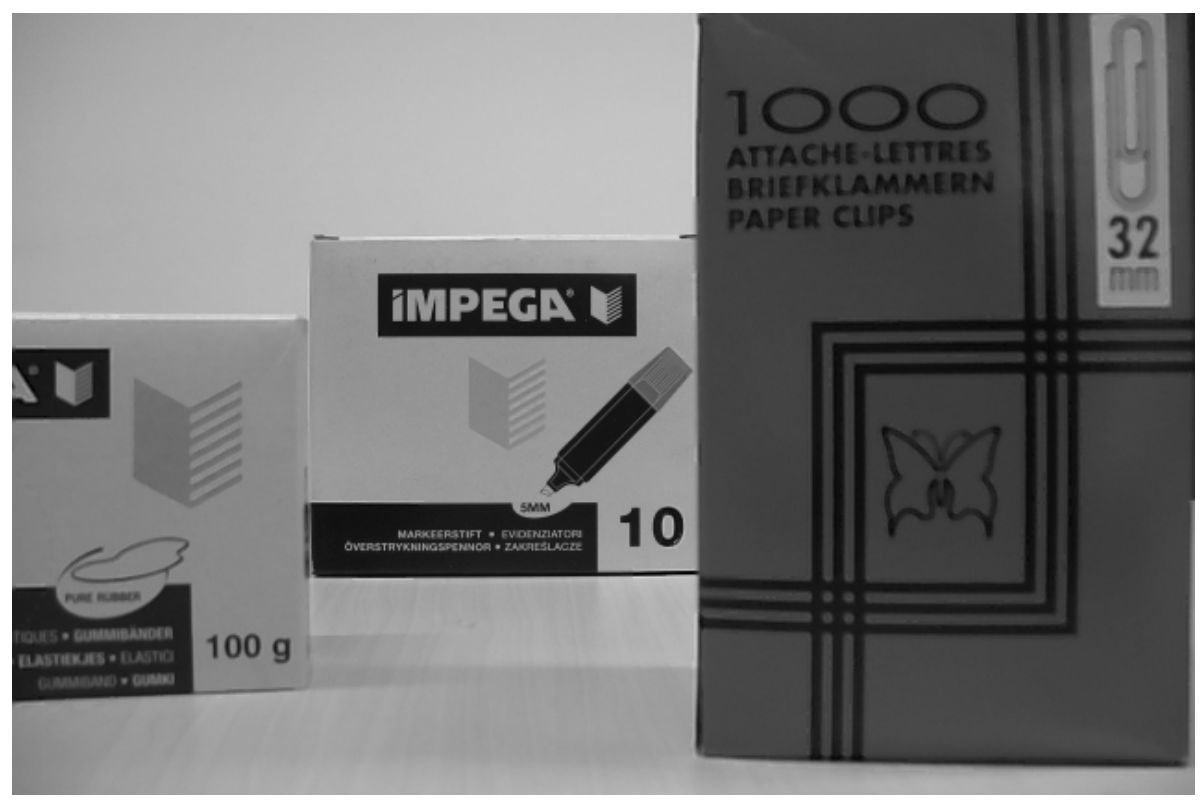

Fig. 7. The restored all-in-focus image 


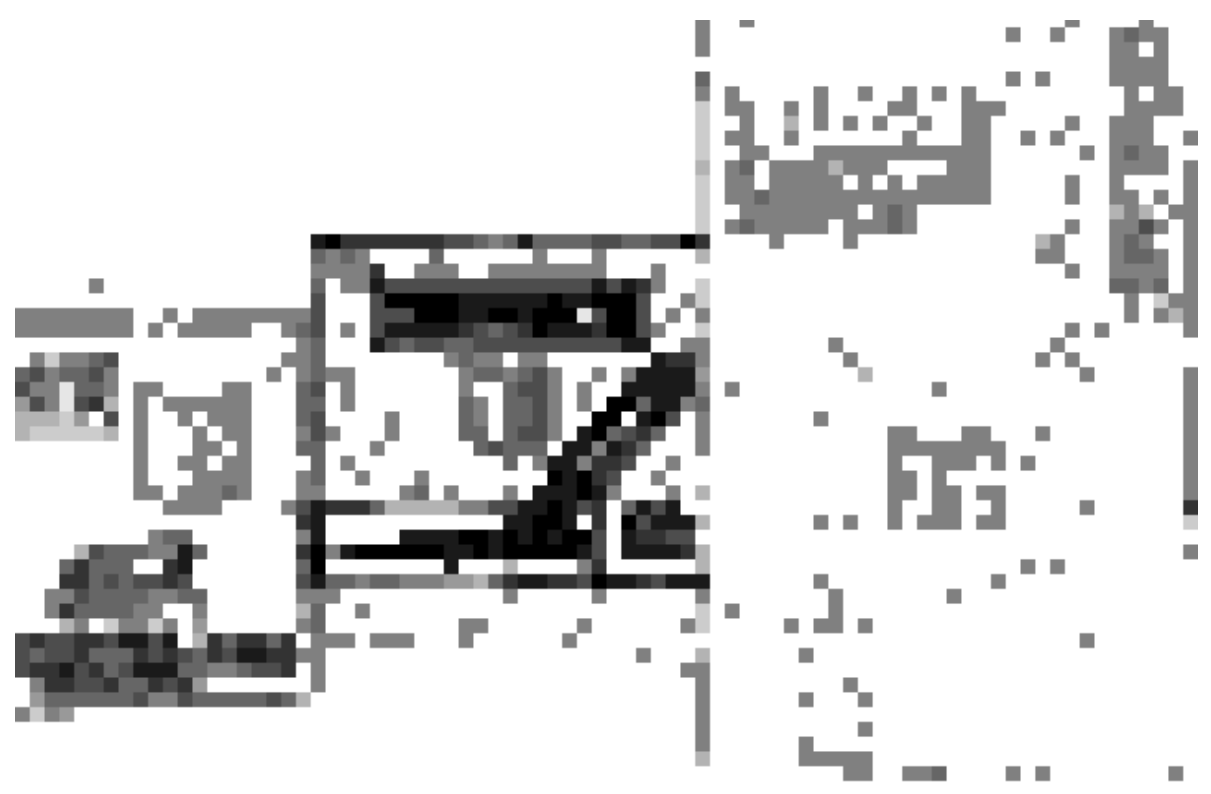

Fig. 8. The blur map obtained by our proposed method: the lighter areas indicate a larger blur radius, while the darker areas indicate a smaller blur radius

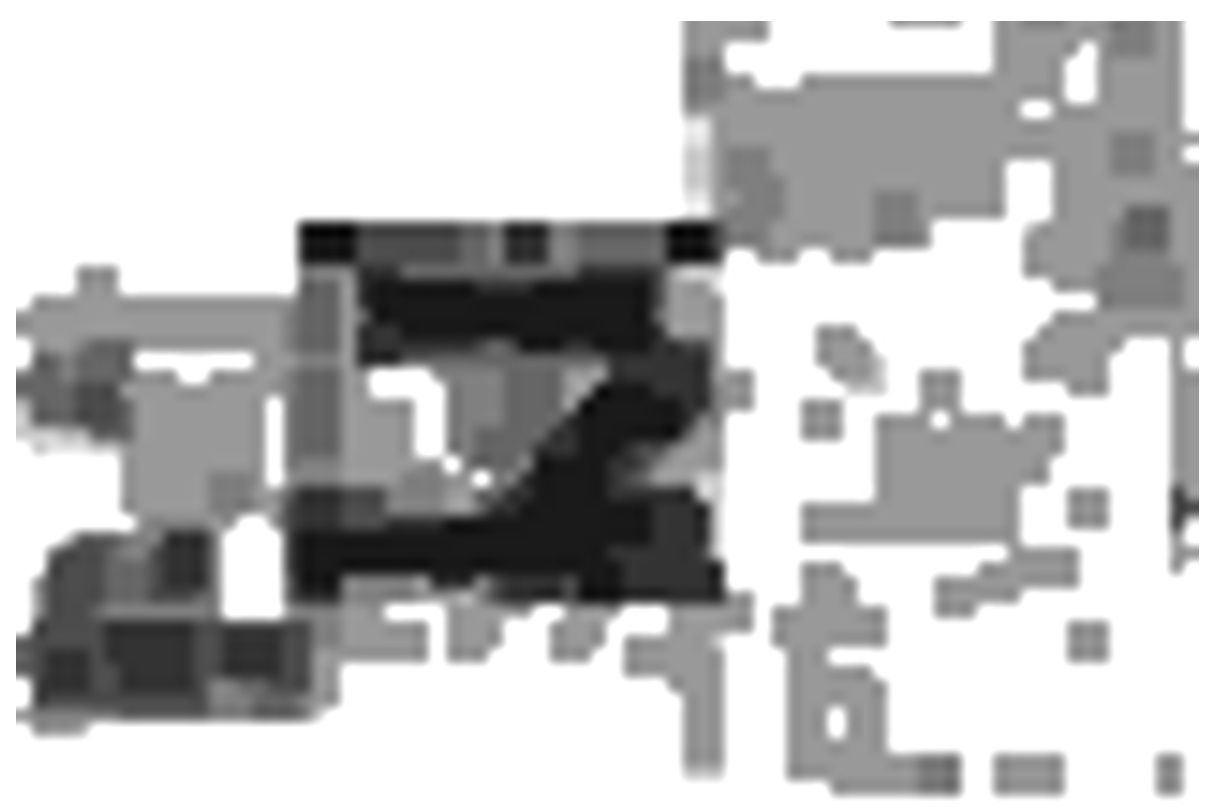

Fig. 9. The final blur map used for adaptive image restoration 


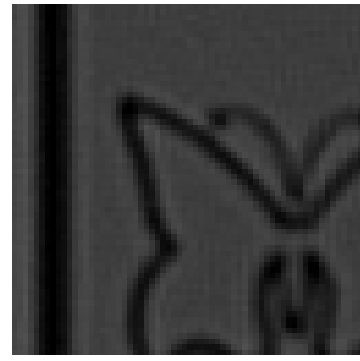

(A)

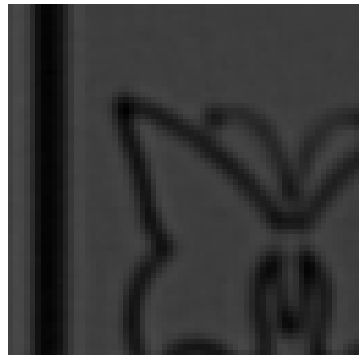

(B)

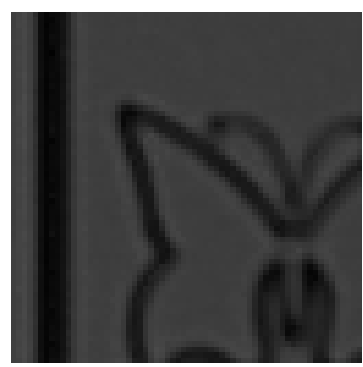

(C)

Fig. 10. Image fragments from the output images using LMS filters with different settings: (A) LMS filters which depend on blur radius only and trained without added noise (B) LMS filters which depend on blur radius only and trained with added noise (C) the proposed LMS filters which depend on both blur radius and image structure and trained with added noise

\section{Conclusion}

We have presented in this paper a novel blur identification and restoration algorithm for a multi-focusing system. The proposed algorithm is based on a robust local blur estimation that uses the difference ratio of a image and its two re-blurred versions. Adaptive least mean square filters, which depend on local image structure and the blur radius, are applied to remove the spatially variant blur and reduce the sensor noise. The proposed method shows promising results for blur identification and restoration given its complexity. Since it does not involve iterations, neither in the blur estimation, nor in the image restoration, it is suitable for real-time applications.

\section{References}

1. Lagendijk, R.L., Biemond, J., Boekee, D.E.: Identification and restoration of noisy blurred image using the expectation-maximization algorithm. IEEE Trans. Acoustic, Speech and Signal Processing 38, 1180-1191 (1990)

2. Elder, J.H., Zucker, S.W.: Local Scale Control for Edge Detection and Blur Estimation. IEEE Trans. Pattern Analysis and Machine Intelligence 20, 699-716 (1998)

3. Kim, S.K., Park, S.R., Paik, J.K.: Simultaneous out-of-focus blur estimation and restoration for digital auto-focusing system. IEEE Trans. Consumer Electronics 34, 1071-1075 (1998)

4. Hu, H., de Haan, G.: Low cost robust blur estimator. In: Proceedings of IEEE Int. Conf. on Image Processing, Atlanta (GA), October 8-11, 2006, pp. 617-620. IEEE Computer Society Press, Los Alamitos (2006) 
5. Hu, H., de Haan, G.: Simultaneous Coding Artifact Reduction and Sharpness Enhancement. In: Proceedings of IEEE Int. Conf. on Consumer Electronics, Las Vegas, pp. 213-214. IEEE Computer Society Press, Los Alamitos (2007)

6. Katsaggelos, A.K.: Iterative image restoration algorithms. Optical Engineering 287, 735-748 (1989)

7. Kondo, T., Fujimori, Y., Ghosal, S., Carrig, J.J.: Method and apparatus for adaptive filter tap selection according to a class, US-Patent: US 6,192,161 B1, February 20, 2001 (2001) 